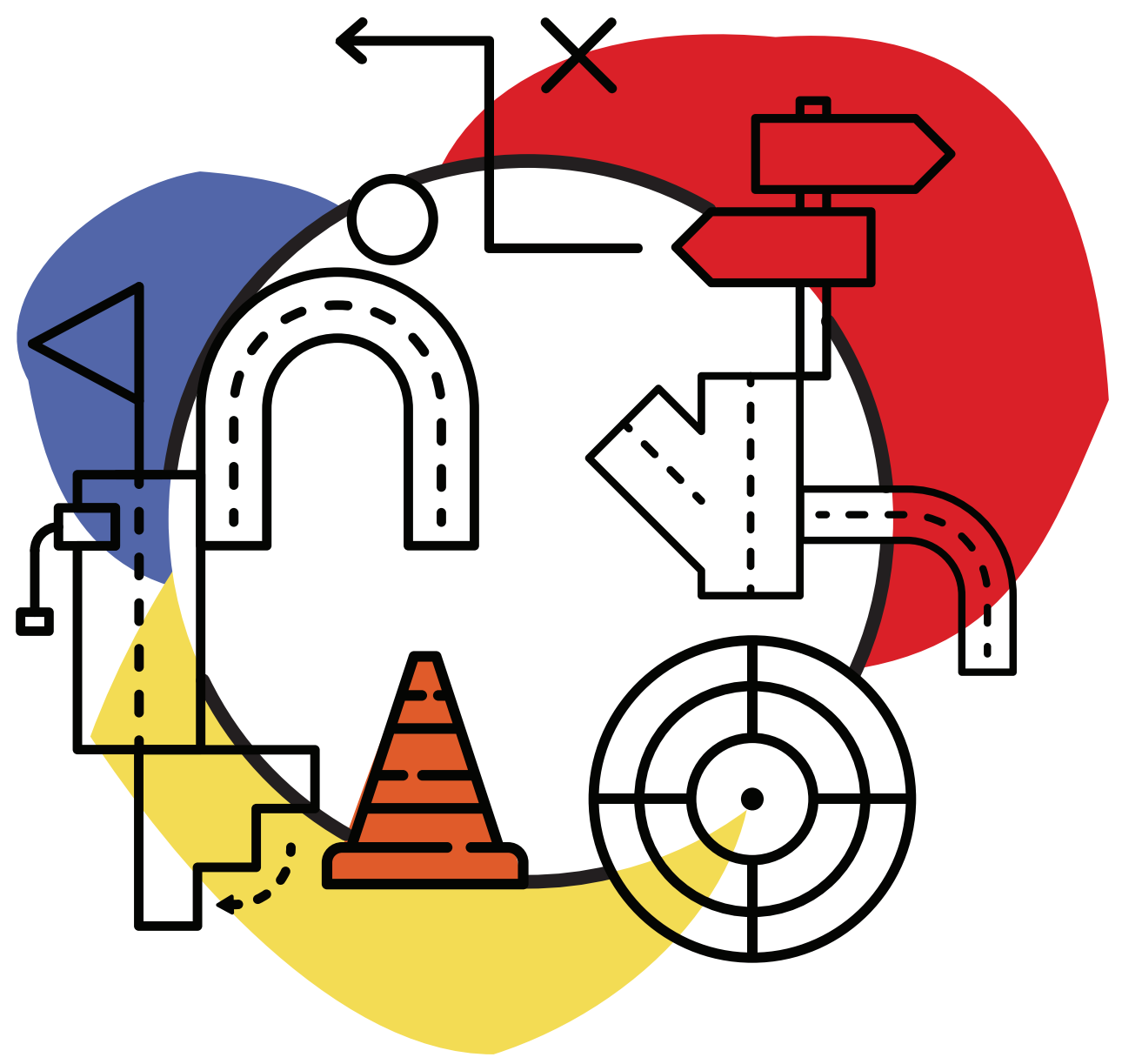

\title{
HERRAMIENTAS PARA LA SISTEMATIZACIÓN DE LA EVALUACIÓN OBJETIVA DE PROYECTOS Y GRADOS DE MADURACIÓN EN INNOVACIÓN EN LAS EMPRESAS
}

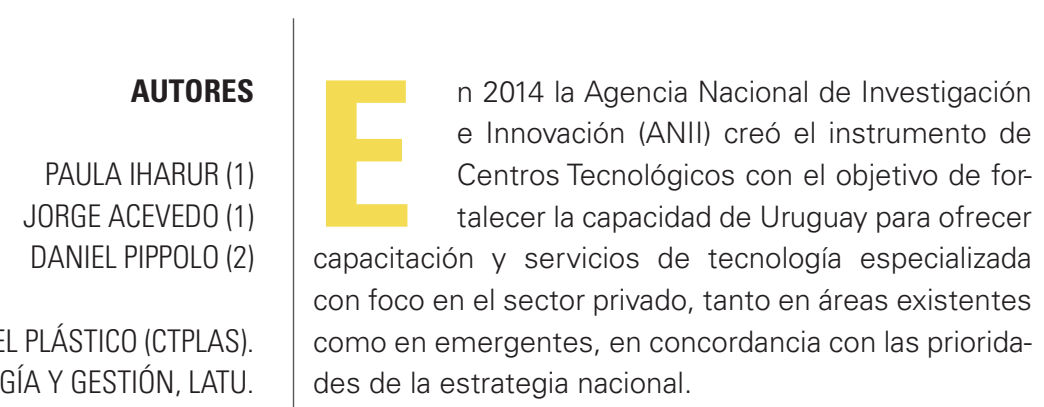

(1) CENTRO TECNOLÓGICO DEL PLÁSTICO (CTPLAS). (2) GERENCIA DE TECNOLOGÍA Y GESTIÓN, LATU. des de la estrategia nacional. 
Los Centros Tecnológicos sectoriales, creados en el marco de este instrumento de ANII, deben tener por objeto contribuir a la mejora de la competitividad de las empresas mediante la generación de conocimiento tecnológico, realizando actividades de I+D+i y desarrollando su aplicación. En este marco emerge como actor el Centro Tecnológico del Plástico (CTplas), que tiene como objetivo implementar soluciones tecnológicas productivas para las empresas del sector y trabajar de forma transversal para lograr una mayor eficiencia de recursos disponibles a nivel local e internacional.

En el entendido de que los Centros Tecnológicos deben permitir establecer nuevas capacidades a nivel país, CTplas aspira a posicionarse como socio estratégico de las empresas en pos de ser un articulador clave entre el sector productivo, la academia y el sector público, agilizando el acceso a la incorporación de mecanismos de innovación en las empresas. Este proceso implica la realización de proyectos conjuntos con empresas, la prestación de servicios de apoyo a la innovación, la capacitación y la transferencia tecnológica.

En esta concepción, la I+D+i es un factor fundamental para la gestión de los Centros, por lo tanto, es mandatorio acompañar y responder con un adecuado diseño organizacional y una infraestructura que pueda soportar la demanda de esta clase de servicios.

\section{Herramientas para clasificar el grado de avance de los proyectos de innovación empresarial}

Para los Centros Tecnológicos resulta clave la incorporación y utilización de herramientas que puedan categorizar las necesidades sectoriales y ordenar la demanda como condicionante para la generación de proyectos.

En esta línea, es esencial la incorporación de herramientas que permitan sistematizar la información obtenida de la realidad de las empresas en contraste con la caracterización de los estadios de innovación en las organizaciones, y abordar integralmente el desarrollo industrial proyectado. Como complemento, se requiere trazar un camino que perfile escalones de avance en materia de I+D+i para las empresas, para poder traducir las ideas a proyectos tangibles, identificar la mejor metodología de acompañamiento a las empresas, medir el esfuerzo en cada etapa y poder visualizar tanto beneficios como costos.
Estas herramientas, además de permitir profundizar el conocimiento de los sectores que los Centros atienden, generan insumos para atender no solo el ámbito industrial al cual se proyectan, sino también a toda la cadena productiva involucrada.

El intercambio de experiencias motivó el desarrollo y la validación de una herramienta específica para evaluar en forma objetiva los proyectos de innovación de empresas. Su aplicación haría posible aumentar la probabilidad de éxito en cuanto a la formulación del proyecto, seleccionar fuentes de financiamiento adecuadas a las realidades de los interesados y definir la mejor estrategia de acompañamiento del CTplas a las empresas solicitantes.

Desde CTplas se profundizó en una de las herramientas utilizadas, sugerida por el Centro Tecnológico español AIMPLAS, que contribuye a medir los niveles de madurez de la tecnología en las organizaciones: los Technology Readiness Levels. Esta herramienta fue adoptada y adaptada por el equipo CTplas como instrumento para la generación y evaluación de proyectos de innovación empresarial.

\section{TRL: Technology Readiness Levels}

Este concepto surge en la NASA para la aplicación en proyectos aeronáuticos o espaciales y, posteriormente, su uso se popularizó para cualquier proyecto. "Los niveles de madurez de la tecnología, más conocidos por sus siglas inglesas originarias TRLs o Technology Readiness Levels, han empezado a usarse en las convocatorias de ayudas del nuevo Programa Marco de Investigación de la Unión Europea (2014-2020), más conocido por H2020" (Ibáñez de Aldecoa Quintana, 2014).

Concretamente, los TRL son una referencia de ayuda para identificar el grado de avance de los proyectos de innovación y facilitan la identificación de elementos para caracterizarlos. A partir de comparar una tecnología bajo la tipificación del nivel de TRL, se pueden inferir características referidas al nivel de madurez en el cual se encuentra. Por lo pronto, esta caracterización favorece la detección de elementos a tener en cuenta para preparar el camino que debe recorrer la empresa para materializar su desarrollo. En esta determinación se puede estudiar y precisar la viabilidad de los proyectos futuros y la menor o mayor cercanía del proyecto al mercado. 


\begin{tabular}{|c|c|c|}
\hline \multicolumn{2}{|r|}{ TRL } & \multirow{2}{*}{$\begin{array}{l}\text { DESCRIPCIÓN } \\
\text { El nivel más bajo de madurez tecnológica. La investigación } \\
\text { científica se comienza a convertir en investigación aplicada y } \\
\text { desarrollo. Los ejemplos pueden incluir investigaciones fun- } \\
\text { damentales y artículos. }\end{array}$} \\
\hline Nivel 1 & $\begin{array}{l}\text { Investigación básica: los princi- } \\
\text { pios elementales son observados } \\
\text { y referidos }\end{array}$ & \\
\hline Nivel 2 & $\begin{array}{l}\text { Investigación aplicada: se formula } \\
\text { el concepto de la tecnología y/o } \\
\text { su aplicación }\end{array}$ & $\begin{array}{l}\text { Una vez que se observan los principios básicos, se formulan } \\
\text { las aplicaciones prácticas. Los ejemplos están limitados a } \\
\text { estudios analíticos y experimentación. }\end{array}$ \\
\hline Nivel 3 & $\begin{array}{l}\text { Función crítica, prueba y estable- } \\
\text { cimiento del concepto }\end{array}$ & $\begin{array}{l}\text { Se inicia la investigación activa y el desarrollo. Los estudios } \\
\text { de laboratorio buscan validar las predicciones analíticas de } \\
\text { los componentes por separado de la tecnología. Los ejem- } \\
\text { plos incluyen componentes que no han sido aún integrados } \\
\text { o no son representativos. }\end{array}$ \\
\hline Nivel 4 & $\begin{array}{l}\text { Análisis de laboratorio del prototi- } \\
\text { po o del proceso }\end{array}$ & $\begin{array}{l}\text { Se lleva a cabo el diseño, desarrollo y análisis de laboratorio } \\
\text { de los componentes tecnológicos. Aquí, los componentes } \\
\text { tecnológicos básicos son integrados para que funcionen jun- } \\
\text { tos. Es un prototipo de "baja fidelidad" en comparación con } \\
\text { el sistema final. }\end{array}$ \\
\hline Nivel 5 & $\begin{array}{l}\text { Análisis de laboratorio del } \\
\text { sistema integrado }\end{array}$ & $\begin{array}{l}\text { Los componentes tecnológicos básicos son integrados con- } \\
\text { juntamente con elementos reales para ser analizados en un } \\
\text { entorno simulado. Este es un prototipo de "alta fidelidad" en } \\
\text { comparación con el sistema final. }\end{array}$ \\
\hline Nivel 6 & Verificación del sistema prototipo & $\begin{array}{l}\text { El prototipo bueno, a partir del nivel } 5 \text {, es analizado en un } \\
\text { entorno relevante. La demostración del sistema o proceso } \\
\text { se lleva a cabo en un entorno operacional. }\end{array}$ \\
\hline Nivel 7 & $\begin{array}{l}\text { Demostración del sistema } \\
\text { piloto integrado }\end{array}$ & $\begin{array}{l}\text { El prototipo está cercano al nivel de sistema operacional } \\
\text { planificado. El diseño final está virtualmente completo. El } \\
\text { objetivo de este nivel es eliminar los riesgos de ingeniería y } \\
\text { manufacturación. }\end{array}$ \\
\hline Nivel 8 & $\begin{array}{l}\text { El sistema incorpora el } \\
\text { diseño comercial }\end{array}$ & $\begin{array}{l}\text { La tecnología ha sido probada para trabajar en la parte final } \\
\text { bajo las condiciones esperadas. En la mayoría de los casos, } \\
\text { este nivel representa el fin del uso de desarrollo de sistemas } \\
\text { verdaderos. }\end{array}$ \\
\hline Nivel 9 & $\begin{array}{l}\text { El sistema está listo para su uso } \\
\text { a escala completa }\end{array}$ & $\begin{array}{l}\text { Aquí, la tecnología adopta su forma final y está lista para su } \\
\text { despliegue comercial. }\end{array}$ \\
\hline $\begin{array}{l}\text { Nivel } \\
\text { posterior al } 9\end{array}$ & Introducción al mercado & $\begin{array}{l}\text { El producto, proceso o servicio se lanza comercialmente en } \\
\text { el mercado y es aceptado por un grupo de clientes (incluido } \\
\text { autoridades públicas). }\end{array}$ \\
\hline
\end{tabular}

Tabla 1. Niveles de maduración tecnológica. Fuente: Consejo Nacional de Ciencia y Tecnología, 2015. 


\section{Incorporación y adaptación de TRL a CTplas}

La incorporación de los TRL surgió a partir de la necesidad de establecer un documento de referencia para la consolidación de los servicios creados y ofrecidos por CTplas. Tomando los TRL y sus dimensiones como punto de partida, se caracterizaron los niveles de innovación y se evaluaron los caminos a seguir para reducir el grado de incertidumbre y las formas de escalarlos de acuerdo a las capacidades para llevar adelante proyectos de innovación en las empresas.

La incorporación y adaptación de los TRL a CTplas implicó un proceso de comprensión y apropiación de la herramienta para adecuarla a las necesidades reales del Centro.

La matriz fue utilizada como referencia para poder estimar los costos del servicio de formulación de proyecto y establecer beneficios para miembros CTplas que lo requirieran, y para determinar el valor agregado de cada servicio ofrecido.

En suma, actualmente esta herramienta se emplea para:

- Valorizar el aporte al diseño del proyecto por parte de CTplas.

- Ser transparentes frente a consultas de empresas miembro CTplas y otras organizaciones a la hora de analizar propuestas y definir caminos a seguir.

- Minimizar los riesgos para las empresas, evitándoles "saltos" muy lejanos entre el desarrollo / innovación y la puesta en el mercado.

\section{La TRL como herramienta en CTplas}

Su proceso de aplicación incluyó las siguientes etapas:

1. Definición de niveles de innovación y categorización de los niveles de innovación. Como se mencionó anteriormente, la tabla TRL alude al grado de avance de los proyectos de I+D+i, del laboratorio al mercado. Por lo tanto, se realizó un estudio minucioso de cada nivel y de sus diferentes ámbitos de observación.

2. Creación de insumos CTplas: adaptación de la herramienta. Para generar un insumo aplicable que lograra abarcar a la gama de servicios que son ofrecidos por CTplas, se incluyó una dimensión anterior a las planteadas por los TRL, el "nivel TRL 0" inte- grado por dos componentes: proyectos primarios y proyectos de transformación. Estos componentes responden a otras necesidades y demandas, e implican otros desafíos para las empresas.

Por lo tanto, en las filas de la planilla de referencia para la evaluación de proyectos confeccionada por CTplas, donde se da respuesta a los diversos escenarios presentados, se identifican tres caracterizaciones de proyectos e ideas: proyectos primarios, proyectos de transformación y proyectos de innovación (TRL).

3. Contenidos y definiciones. La incorporación de los niveles complementarios -proyectos primarios y proyectos de transformación- implicó su establecimiento y caracterización para poder aplicar la herramienta de forma correcta y replicarla ante diversos escenarios.

Como forma de acompañar y seguir la línea propuesta por los TRL, se caracterizaron los proyectos primarios y proyectos de transformación incorporando una definición para cada uno. Estas definiciones fueron elaboradas cumpliendo un proceso de pruebas y validación y contemplando aspectos para evaluar el entorno de ejecución del proyecto y su nivel de complejidad.

Como resultado se construyó la siguiente caracterización:

a. Proyectos primarios: Proyectos de mejora o resolución de problemas en el ámbito de la empresa. Focalizados en temas específicos, en un entorno real y con una complejidad baja, implican, por ejemplo, la incorporación de expertos para solucionar problemas puntuales en la empresa.

b. Proyectos de transformación: son proyectos de complejidad media que suponen el desarrollo y aplicación de estrategias y la articulación entre diversos actores para la resolución de problemas. Por tanto, implican la resolución de problemas de mayor alcance en un entorno real, y pueden abarcar, por ejemplo, la formulación de proyectos, los análisis financieros, el relevamiento de presupuestos, la búsqueda de proveedores tecnológicos, etcétera.

4. Detección de fuentes de financiamiento. En su rol de articulador, CTplas busca generar sinergias 
entre las capacidades de las empresas nacionales del plástico, considerando la demanda del entorno y apostando al fomento de nuevos negocios por medio de la utilización de las fuentes de financiamiento disponibles. Por este motivo también se incorporaron en la herramienta de caracterización de los proyectos los diferentes instrumentos de financiamiento disponibles para acompañar a cada proyecto en sus diversos niveles.

A continuación se enumeran las principales estrategias que ha promovido y aplicado el CTplas hasta la fecha:

- Pasante FING. Esta propuesta de Facultad de Ingeniería involucró a un estudiante avanzado de Ingeniería Química o Mecánica para el trabajo en un proyecto de mejora en su empresa. Como asignatura de final de carrera, propone que los estudiantes enfrenten y resuelvan problemas reales de las empresas, buscando formas de mejorar y optimizar los procesos. Su denominación como pasantía-trabajo experimental contempla el formato de trabajo no rutinario en una empresa formal radicada en el país.

- Experto SES. A través de la Cámara de Comercio Uruguayo Alemana, expertos alemanes del Senior Experten Service (SES; fundación de la industria alemana para la cooperación internacional) realizan asesorías en empresas. CTplas gestiona las solicitudes ante la Cámara en caso de que las empresas manifiesten interés en contar con la asesoría de un profesional de primer nivel.

- Circulación de talentos - herramienta de la Agencia Nacional de Investigación e Innovación (ANII). Permite la partida de técnicos para realizar una formación en el exterior o la visita de técnicos del extranjero.

- Fondo Industrial. Es un instrumento del Ministerio de Industria, Energía y Minería (MIEM) que otorga fondos no reembolsables. Su objetivo es diversificar y tecnificar la estructura productiva nacional, así como desarrollar las industrias de soporte que potencien la competitividad de las cadenas de valor existentes.

- PIEP (Proyecto de Internacionalización de la Especialización Productiva). Es un instrumento del MIEM propuesto en el marco del Fondo para la Convergencia Estructural del Mercosur (FOCEM). Convoca a empresas uruguayas a presentar proyectos de inversión en industria y servicios conexos que incorporen tecnologías innovadoras, que presenten vínculos con la región y cuyo foco comercial esté dirigido a mercados del exterior.

Por último, se identifican las diversas herramientas de ANII para promover la innovación e investigación en sus diversas etapas y modalidades:

- Herramientas para la innovación: El objetivo de esta línea es apoyar a las empresas para que incrementen y fortalezcan sus capacidades internas para innovar y de esa forma se encuentren mejor preparadas para diseñar e implementar sus proyectos de innovación. - Implementación de la innovación: El objetivo de esta línea es apoyar a las empresas para que implementen proyectos de innovación, ya sea en productos (bienes y servicios), en procesos, en organización o en comercialización, y de esa manera puedan aumentar su productividad y su competitividad.

- Potenciar la innovación: El objetivo de esta línea es apoyar a las empresas para que desarrollen y fortalezcan sus proyectos de innovación que hayan resultado técnicamente exitosos, incluyendo la realización de pruebas piloto, la protección intelectual y la profundización de sus vinculaciones con los mercados de destino.

\section{Conclusiones}

A meses de implementada la herramienta en CTplas, se destaca que es un elemento de fácil aplicación que ayuda a caracterizar rápidamente las ideas y proyectos más concretos y es de utilidad para su conceptualización. Además, ayuda a minimizar riesgos -considerando alternativas de puesta en el mercado- y costos del proyecto.

Asimismo, otorga transparencia de gestión del Centro para la definición de los servicios. Según el grado de dificultad del proyecto, permite determinar el valor agregado de CTplas y estimar con mayor exactitud los recursos requeridos para dar andamiaje a una propuesta.

También se destaca como una herramienta que ayuda a estandarizar y categorizar los proyectos en otros centros y organizaciones, y los inversores necesarios para su desarrollo. Así contribuye a definir o alcanzar una idea primaria del riesgo que la propuesta implica.

La adopción de esta herramienta en organizaciones en general facilitaría la coordinación y articulación para proyectos asociativos, ya que se puede apreciar con 
mayor claridad los esfuerzos que se deberían dedicar para la generación de capacidades entre los actores involucrados. Desde CTplas se encuentra una oportunidad para trabajar de forma más coordinada entre los centros y organizaciones, y alinear así los esfuerzos en pos de la promoción y generación de capacidades para los diversos sectores productivos.

\section{Referencias}

Consejo Nacional de Ciencia y Tecnología, 2015. Convocatorias SE CONACYT, Innovación tecnológica. Anexo 1: Niveles de maduración tecnológica [En línea]. México DF: Gobierno de la República. [Consulta: 5 de octubre de 2017]. Disponible en: http://conacyt.gob. $\mathrm{mx} / \mathrm{index}$.php/resultados-extranjero/convocatorias-conacyt/convocatorias-fondos-sectoriales-constituidos/ convocatoria-se-conacyt-innovacion-tecnologica/convocatorias-cerradas-se-conacyt-innovacion-tecnologica/ convocatoria-se-conacyt-innovacion-tecnologica-2015/9282-anexo-1-niveles-de-maduracion-tecnologica/file

Ibáñez de Aldecoa Quintana, Juan Miguel, 2014. Niveles de madurez de la tecnología. Technology readiness levels. trls. Una introducción [En línea]. Madrid: Ministerio de Industria. [Consulta: 23 de junio de 2017]. En: Revista Economía Industrial, 393, pp.165-170. Disponible en: http://www.minetad.gob.es/Publicaciones/ Publicacionesperiodicas/Economialndustrial/RevistaEconomialndustrial/393/NOTAS.pdf

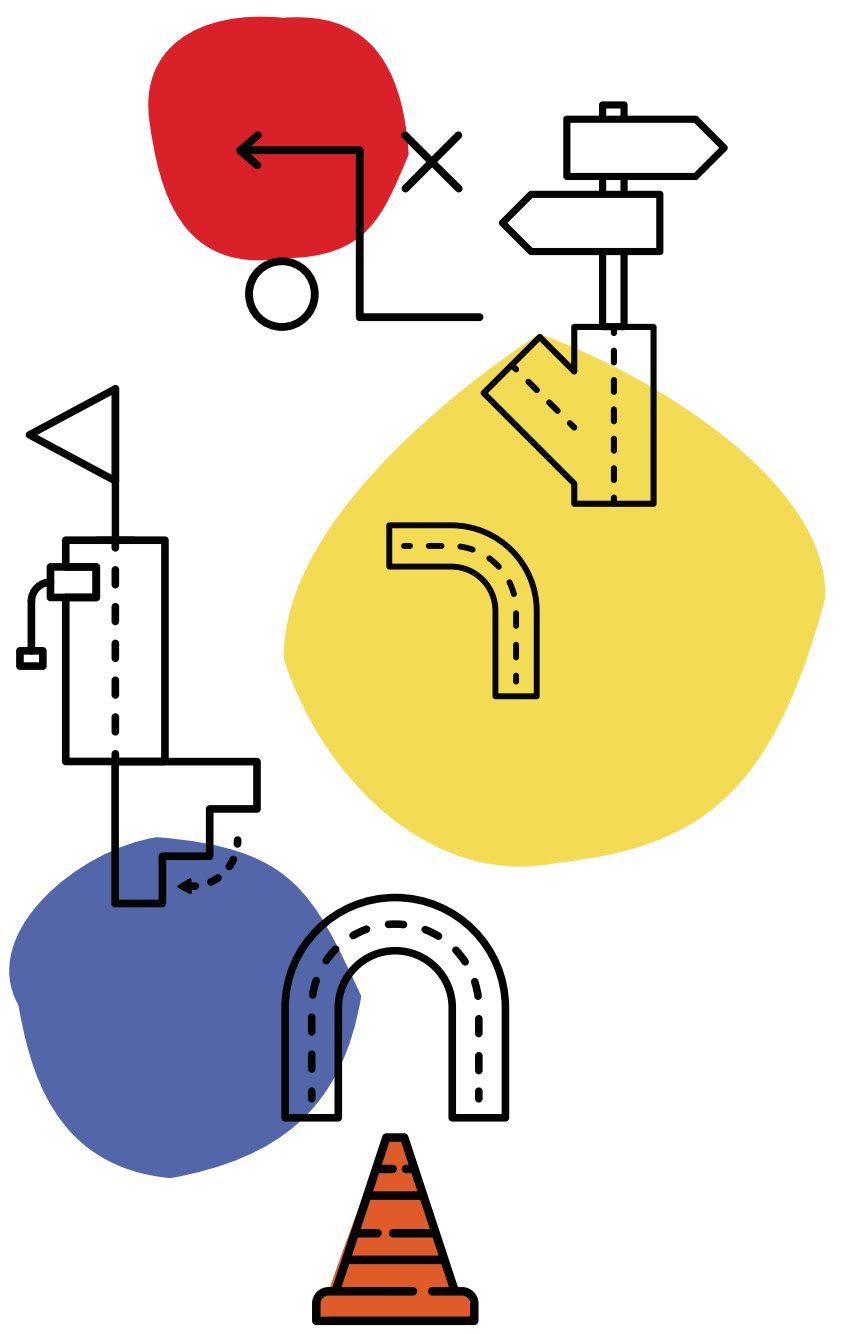




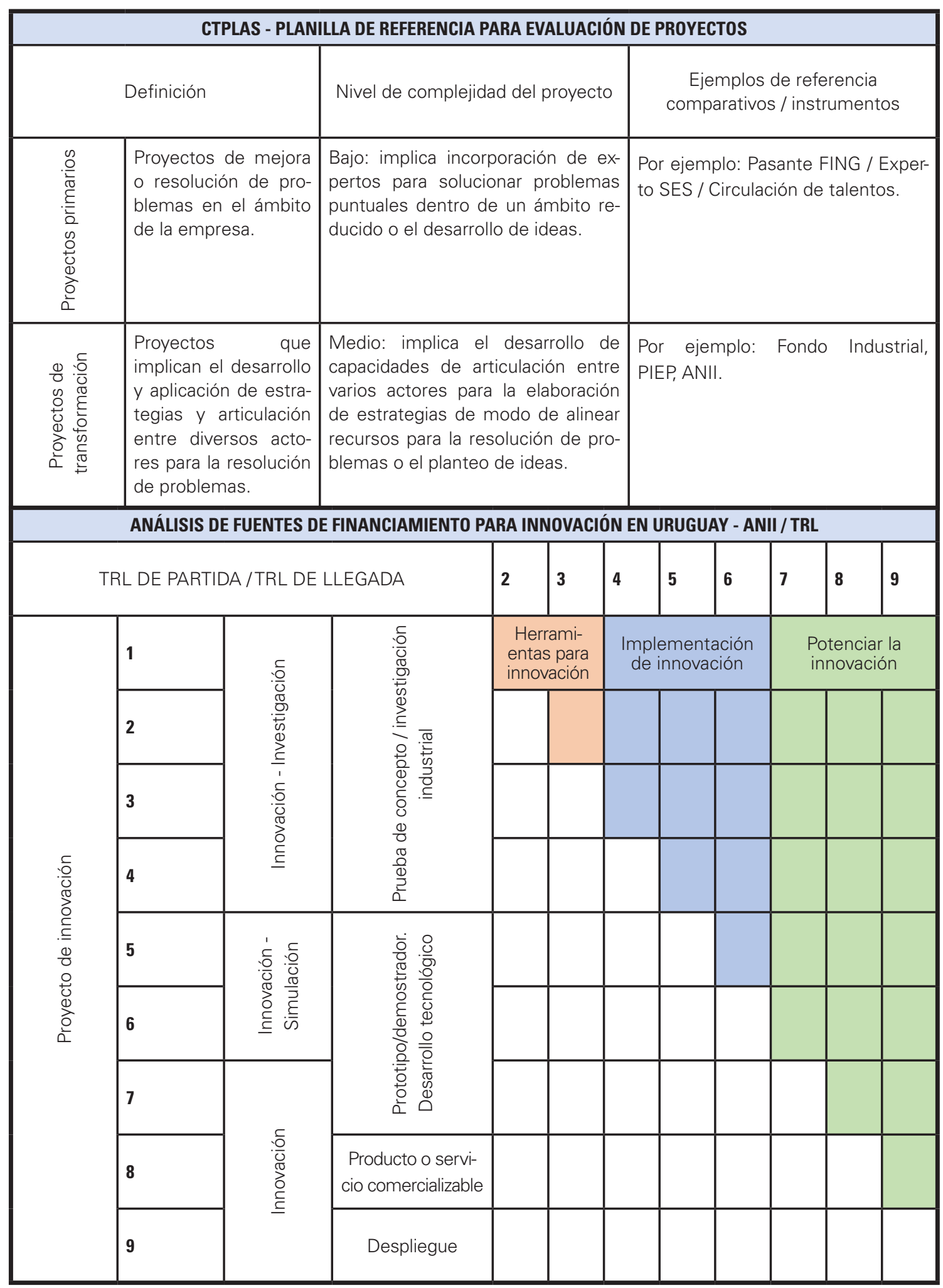

Tabla 2. MatrizTRL. 